\title{
Desenvolvimento juvenil de Cyrtograpsus angulatus Dana (Crustacea, Decapoda, Grapsidae), em laboratório
}

\author{
Paulo Juarez Rieger ${ }^{1}$ \\ Roberta Beltrão ${ }^{2}$
}

\begin{abstract}
Juvenile development of Cyrtograpsus angulatus Dana (Crustacea, Decapoda, Grapsidae), in laboratory. This paper describes the first nine juvenile stages of Cyrtograpsus angulatus Dana, 1851 obtained in laboratory conditions from three ovigerous females collected at "Molhes da Barra", Rio Grande, Brazil $\left(32^{\circ} \mathrm{S}\right.$ and $\left.52^{\circ} \mathrm{W}\right)$. The main morphological features, the secondary sexual characters and the gills ontogeny were observed. Twenty one individuals reached until the ninth juvenile stage. Sexual dimorphism can be verified from the fourth juvenile stage and the number of gills are complete at the second juvenile stage.

KEY WORDS. Crustacea, Brachyura, Grapsidae, development, juvenile
\end{abstract}

A família Grapsidae encontra-se representada no litoral do Rio Grande do Sul, por seis espécies das sub-famílias Sesarminae, Varuninae e Grapsinae as quais estão distribuídas em quatro gêneros: Chasmagnathus granulata Dana, 1851; Armases rubripes (Rathbun, 1914); Cyrtograpsus altimanus Rathbun, 1914; Cyrtograpsus angulatus Dana, 1851; Cyrtograpsus affinis Dana, 1851 e Pachygrapsus transversus Gibbes, 1850.

Cyrtograpsus angulatus apresenta distribuição geográfica compreendida entre, Atlântico Sul Ocidental: Brasil (do Rio de Janeiro ao Rio Grande do Sul), Uruguai e Argentina (incluindo a Patagônia). Pacífico Oriental: Peru e Chile. Costumam formar grandes concentrações na zona entre-marés, em profundidades muito pequenas. É encontrado também sobre a vegetação das praias (MELO 1996).

Os estudos envolvendo larvas de Crustáceos Decápodos tiveram seu início em meados do século passado. THOMPSON (1903) em seu estudo, lançou as bases para os estudos sobre desenvolvimento. É considerado ponto comum entre os carcinologistas, a importância de se conhecer os estágios correspondentes à fase juvenil, quando os indivíduos começam a desenvolver os caracteres sexuais secundários, além de apresentar características diferentes às dos adultos. Todavia, existe uma escassez de trabalhos sobre desenvolvimento juvenil, devido provavelmente a dificuldade de se manter um cultivo até a fase juvenil e, nesta, até que o dimorfismo sexual ocorra, uma vez que o tempo necessário é muitas vezes longo. Portanto, muitos autores detêm seus estudos somente ao desenvolvimento larval.

1) Laboratório de Zoologia de Crustacea Decapoda, Departamento de Ciências Morfológicas e Biológicas, Fundação Uniersidade do Rio Grande. Caixa Postal 474, 96201-900 Rio Grande, Rio Grande do Sul, Brasil. E-Mail: dmbpjr@super.furg.br

2) Bolsista de Iniciação Científica (FAPERGS). E-Mail: rbeltrao@yahoo.com 
Inicialmente, um dos problemas enfrentados no cultivo de larvas em laboratório, era a alimentação, a qual constituía-se de plâncton e muitas vezes não surtia efeitos satisfatórios, pois, em muitos casos, os componentes do zooplâncton se alimentavam das larvas.

Os primeiros trabalhos visando o desenvolvimento juvenil sofreram um impulso com a melhora das técnicas de alimentação, por meio do aprimoramento das técnicas de cultivo de Artemia sp..

Na Família Grapsidae só se conhece o primeiro estágio juvenil das seguintes espécies:, Armases rubripes, Sesarma ricordi H. Milne Edwards por DIAS \& EWALD (1968), Sesarma perracae por LAM (1969), Sesarma (Holometopus) dehaani Randall, 1840 por BABA \& MIYATA (1971), Helice tridens Rathbun por BABA \& MORIYAMA (1972), Chiromantes bidens de Haan por FUKUDA \& BABA (1976), Sesarma (Holometopus) rectum Randall, 1840 por FRANSOZO (1986), Percnon gibbesi H. Milne Edwards, 1853 por Paula \& Hartnoll (1989) Percnon abbreviatum Dana, Percnon guinotae Crosnier, Percnon planissimum Herbst por HARTNOLL (1992), Brachynotus atlanticus Forest, 1957 por RODRIGUEZ et al. (1992), Chasmagnathus granulata por RIEGER \& NAKAGAWA (1995) e Sesarma curacaoense Rathbun, 1897 por ANGER et al. (1995) e Pachygrapsus transversus por Flores et al. (1998). Destes, somente Sesarma (Holometopus) rectum, Chasmagnathus granulata e Pachygrapsus transversus estudados respectivamente por Fransozo (1986), RiEger \& NAKAGAWA (1995) e FlorES et al. (1998), tiveram seus estudos sobre o desenvolvimento juvenil estendidos até o estabelecimento dos caracteres sexuais secundários.

Pode-se dizer que cerda é uma extensão cutícular, provida de uma base articulada definida (INGLE 1992). O estudo das cerdas em juvenis de braquiúros tem se limitado à sua contagem nos apêndices, e ao mapeamento da sua distribuição ao longo do corpo do animal. Em Anomura, podemos citar o trabalho sobre os primeiros estágios juvenis de Aegla prado Schmitt, 1942 (BOND-BUCKUP et al. 1996). Já com relação a fase larval destacam-se os trabalhos de POHLE \& TELFORD (1981) e INGLE (1992), no qual foram reconhecidas mais de 30 tipos diferentes de cerdas com a utilização de microscopia eletrônica. Existe uma grande dificuldade em se determinar a diferença entre determinados tipos de cerda utilizando a microscopia ótica, portanto nos trabalhos que utilizam apenas esse tipo de recurso pode-se fazer apenas um levantamento não muito aprofundado dos diferentes tipos de cerdas. Sobre a morfologia de cerdas, destacam-se, além dos citados acima, THOMAS (1970), Fish (1972), FARMER (1974), DRACH \& JACQUES (1977a,b), FACTOR (1978), KUNZE \& ANDERSON (1979), SCHEMBRI (1982), WATLING \& THISTLE (1989) e JACQUES et al. (1989).

\section{MATERIAL E MÉTODOS}

As coletas foram realizadas quinzenalmente durante um ano, sendo efetuadas as medidas de temperatura e salinidade, no local das coletas.

As fêmeas ovígeras de Cyrtograpsus angulatus foram coletadas, junto às rochas, na Prainha próxima à região dos Molhes da Barra de Rio Grande, Rio Grande do Sul. O transporte foi efetuado em caixas térmicas, com uma pequena quantidade de água e substrato dos locais de coleta. 
No laboratório, as fêmeas ovígeras foram mantidas isoladas, em aquários com capacidade para 20 litros contendo água do mar filtrada, com aeração, até a eclosão.

Iniciada a eclosão, as larvas foram observadas para a verificação da possível ocorrência de um estágio de pequena duração, denominado pré-zoea. Poucas horas após a eclosão, as zoea I, foram concentradas com auxílio de um foco de luz e individualizadas com um conta-gotas em pequenos recipientes com capacidade para $20 \mathrm{ml}$ de água do mar filtrada.

Foram realizadas três observações controladas (denominadas A, B e C), envolvendo um total de 260 larvas, oriundas de três fêmeas ovígeras. Os cultivos foram realizados em câmara climática na ausência de luz.

Observação controlada A: realizada em câmara climática, com temperatura de $23,5^{\circ} \mathrm{C} \pm 2^{\circ} \mathrm{C}$ e água do mar com salinidade de $34 \%$. Foram individualizadas 60 larvas.

Observação controlada B: realizada nas mesmas condições de salinidade e temperatura que a observação controlada A. Foram individualizadas 100 larvas.

Observação controlada C: realizada em câmara climática, com temperatura de $22,5^{\circ} \mathrm{C} \pm 2^{\circ} \mathrm{C}$ e salinidade de $30 \%$. Foram individualizadas 100 larvas.

Os cultivos somente eram retirados da câmara climática por ocasião das observações diárias, para constatação da mortalidade e verificação da ocorrência de ecdises, com a conseqüente determinação das mudanças de estágio acompanhadas da troca da água e introdução do alimento - algas microscópicas Tetraselmis sp., Brachionus plicatillis Müller, 1786 (Rotifera) e "nauplii" recém eclodidos de Artemia sp. para os estágios da fase larval. No estágio juvenil, os indivíduos foram alimentados com "nauplii" de Artemia sp. e pedaços de músculo de peixe.

Os juvenis mortos e as exúvias de cada estágio foram fixadas e preservadas em uma mistura de álcool etílico $96 \%$ e glicerina na proporção de 1:1.

As descrições morfológicas, medidas e desenhos foram efetuados a partir de exúvias e juvenis preservados, sob microscópio óptico Olimpus BX 40, com câmara clara. Os desenhos das cerdas foram efetuados com indivíduos do oitavo estágio juvenil.

$\mathrm{Na}$ apresentação dos resultados, tabelas e figuras, dos diferentes estágios, foram adotados os seguintes símbolos: (JI) $1^{\circ}$ estágio juvenil; (JII) $2^{\circ}$ estágio juvenil; (JIII) $3^{\circ}$ estágio juvenil; (JIV) $4^{\circ}$ estágio juvenil; (JV) $5^{\circ}$ estágio juvenil; (JVI) $6^{\circ}$ estágio juvenil; (JVII) $7^{\circ}$ estágio juvenil; (JVIII) $8^{\circ}$ estágio juvenil; (JIX) $9^{\circ}$ estágio juvenil; (JX) $10^{\circ}$ estágio juvenil; (JXI) $11^{\circ}$ estágio juvenil; (JXII) $12^{\circ}$ estágio juvenil e (JXIII) $13^{\circ}$ estágio juvenil; (cp) cerda plumosa; (cps) cerda plumosa com inserção supracuticular; (cpp) cerda pouco plumosa do ante-penúltimo segmento da antena; (cl) cerda lisa; (cpd) cerda plumodenticulada; (cpdm) cerda plumodenticulada com dentículos modificados em forma de bigorna "anvio-shaped denticules"; (ca) cerdas simples com "annulus"; (css) cerda serrilhada sem "annulus" e com inserção supracuticular; (csa) cerda serrilhada com "annulus"; (csc) cerda serrilhada com forma "cuspidate"; (csa) cerda serrilhada com "annulus".

Foram analisados 10 indivíduos de cada estágio. Na descrição dos estágios, os valores entre parênteses, são os que apareceram em menor frequiência. Os juvenis 
e/ou as exúvias encontram-se depositadas na coleção do Laboratório de Zoologia de Crustáceos Decápodes do Departamento de Ciências Morfobiológicas da Fundação Universidade do Rio Grande, com os lotes: 119-137, 275, 277, 284, 285-288, 292, 295, 547 e 548 .

A metodologia utilizada baseou-se em RiEGER \& NAKAGAWA (1995). A terminologia geral adotada seguiu as de, FRANSOZO (1986), INGLE (1992) e RIEGER \& NAKAGAWA (1995), enquanto para a nomenclatura das cerdas, WATLING \& THISTLE (1989).

\section{RESULTADOS E DISCUSSÃO}

Nos Molhes da Barra de Rio Grande e arredores, constatou-se a ocorrência de fêmeas ovígeras de Cyrtograpsus angulatus durante o período compreendido entre os meses de setembro a meados de abril.

Para eclosão de larvas, em laboratório, os resultados mais viáveis foram aqueles obtidos a partir de fêmeas ovígeras com embriões em estágio mais avançado de desenvolvimento, caracterizado pela posse de olhos compostos pigmentados, facilmente observáveis através da membrana envoltória do ovo.

O desenvolvimento larval é constituído de cinco estágios na fase zoea e um estágio na fase megalopa (RIEGER \& VIEIRA 1997).

Para o desenvolvimento juvenil o melhor resultado foi observado no experimento $\mathrm{C}$, com a sobrevivência de 19 indivíduos até o nono estágio, e três até o décimo terceiro. Os resultados de duração e sobrevivência de cada estágio juvenil estão representados na tabela I.

O dimorfismo sexual foi observado a partir do estágio JIV, com a definição de quatro pares de pleópodos $\left(\mathrm{Pl}_{2} \mathrm{a} \mathrm{Pl}_{5}\right)$ para as fềmeas e dois pares $\left(\mathrm{Pl}_{1}\right.$ e $\left.\mathrm{Pl}_{2}\right)$ para os machos.

Tabela I. Cyrtograpsus angulatus. Resultados do cultivo em diferentes experimentos (A, B, C). (média) Duração média acumulada em dias, (D e D') duração mínima e máxima, (n) número de indivíduos vivos, (M) número de indivíduos mortos, (S\%) porcentagem de sobrevivência no estágio.

\begin{tabular}{llllllllllllll}
\hline Experimento & JI & JII & JII & JIV & JV & JVI & JVII & JVIII & JIX & JX & JXI & JXII & JXIII \\
\hline Média & 30,83 & 34,74 & 40,22 & 47,90 & 63,65 & 107,00 & 157,90 & 180,40 & & & & \\
D & 27 & 32 & 36 & 43 & 55 & 75 & 121 & 161 & & & & \\
A D' & 33 & 38 & 42 & 50 & 70 & 132 & 181 & 192 & & & & & \\
n & 31 & 31 & 31 & 31 & 31 & 31 & 31 & 17 & 0 & 0 & 0 & 0 & 0 \\
M & 0 & 0 & 0 & 0 & 0 & 0 & 0 & 14 & 17 & 0 & 0 & 0 & 0 \\
S\% & 100,00 & 100,00 & 100,00 & 100,00 & 100,00 & 100,00 & 100,00 & 54,83 & 0 & 0 & 0 & 0 & 0 \\
\hline Média & 31,77 & 35,88 & 40,49 & 47,41 & 60,64 & 103,20 & 153,50 & 178,90 & & & & & \\
D & 28 & 31 & 38 & 45 & 53 & 75 & 117 & 162 & & & & & \\
B D' & 40 & 50 & 54 & 75 & 96 & 136 & 181 & 189 & & & & \\
n & 63 & 60 & 57 & 53 & 50 & 47 & 44 & 21 & 0 & 0 & 0 & 0 & 0 \\
M & 3 & 3 & 3 & 4 & 3 & 3 & 3 & 23 & 21 & 0 & 0 & 0 & 0 \\
S\% & 95,45 & 95,23 & 95,00 & 92,98 & 94,33 & 94,00 & 93,61 & 47,72 & 0 & 0 & 0 & 0 & 0 \\
\hline Média & 40,22 & 44,43 & 50,22 & 57,39 & 68,52 & 83,04 & 111,90 & 148,00 & 199,70 & 244,20 & 269,90 & 286,30 \\
D & 35 & 39 & 45 & 51 & 62 & 74 & 95 & 120 & 157 & 203 & 253 & 282 & \\
D' & 49 & 57 & 66 & 78 & 99 & 125 & 167 & 204 & 244 & 273 & 289 & 290 & \\
n & 48 & 48 & 48 & 48 & 48 & 48 & 48 & 34 & 15 & 9 & 7 & 3 \\
M & 0 & 0 & 0 & 0 & 0 & 0 & 0 & 14 & 19 & 6 & 2 & 4 \\
S\% & 100,00 & 100,00 & 100,00 & 100,00 & 100,00 & 100,00 & 100,00 & 70,83 & 44,11 & 60,00 & 77,77 & 42,85 & 0 \\
\hline
\end{tabular}




\section{Primeiro estágio juvenil}

Carapaça (Fig. 1a) dorsalmente convexa, com comprimento aproximadamente igual à largura, dando-lhe um formato quadrangular. Apresenta dois pares de espinhos, sendo um par situado na região ântero-lateral e um par na região mediana. Presença de cerdas lisas e plumosas (ver figura 4 para cerdas) distribuídas por toda a carapaça. Não apresentam fronte saliente; olhos pedunculados bem desenvolvidos (Fig. 1a). Abdômen formado por seis somitos distintos, mais largos do que longos, revestidos por cerdas lisas e plumosas. Telso com a margem posterior convexa e com cerdas lisas e plumosas marginais e dorsais (Fig. 1b). Apresentam quatro pares de pleópodos (Fig. $5 \mathrm{JI})$ : primeiro $\left(\mathrm{Pl}_{2}\right)$ e segundo par $\left(\mathrm{Pl}_{3}\right)$ apresentam um bulbo na parte interna com uma cerda apical, terceiro par $\left(\mathrm{Pl}_{4}\right)$ com bulbo mas sem cerda e quarto par $\left(\mathrm{Pl}_{5}\right)$ desprovido de bulbo, distribuídos do segundo ao quinto somito abdominal, diminuindo de tamanho nesta ordem. Apresentam um par de urópodos localizados no sexto somito abdominal (Fig. 5). Os quelípodos (Fig. 1cQ), são semelhantes, com dois pares de artrobrânquias, e segmentos providos de cerdas lisas e plumosas. Os dedos, fixo e móvel, apresentam pequenos dentes na margem interna. Os segundos pereiópodos semelhantes aos demais na estrutura geral, com cerdas lisas e plumosas, com um par de pleurobrânquias. Terceiro e quarto pereiópodo semelhante na estrutura geral, mas sem brânquia no terceiro (Fig. 1c, $\mathrm{P}_{3}$ e $\mathrm{P}_{4}$ ). Portanto diferindo do adulto no que diz respeito ao número de brânquias.

Antênula (Fig. 2a). Segmento basal não muito desenvolvido desprovido de cerdas. Pedúnculo bi-segmentado contendo cerdas lisas. O endopodito é bi-segmentado, desprovido de cerdas no segmento proximal, e com três ou quatro cerdas lisas no segmento distal, sendo duas terminais. O exopodito é tetra-segmentado, sem cerdas ou estetos no primeiro segmento, de três a cinco estetos no segundo, de quatro a seis estetos e uma cerda lisa no terceiro, e quatro ou cinco estetos no quarto segmento.

Antena (Fig. 2b). Dividida em 10 (9) segmentos com cerdas lisas e plumosas distribuídas da seguinte maneira: 5, 2 (1), 3 (2), 0, 4 (0), 4 (1), 5 (1), 5 (3), 3 (0), 3 (2).

Mandíbula (Fig. 2c). Apresenta uma lâmina cortante e um palpo tri-segmentado, com entre 9 e 13 cerdas plumosas.

Maxílula (Fig. 2d). Endopodito bi-segmentado, com duas cerdas lisas no segmento proximal e 6 (5) cerdas lisas e plumosas no lóbo distal. Endito coxal com 24 (23) cerdas lisas, cúspides (WATLING \& THISTLE 1989), plumosas, serradas e plumodenticuladas, e endito basal de 25 a 30 cerdas lisas, plumosas, cúspides, serradas e plumodenticuladas.

Maxila (Fig. 2e). Lobo proximal do endito coxal com 18 ou 19 cerdas lisas e plumosas, lobo distal do endito coxal com 7 (8) cerdas lisas e plumosas, lobo proximal do endito basal de 10 a 13 cerdas lisas e plumosas, lobo distal do endito basal de 12 a 14 cerdas lisas e plumosas. Endopodito 2 (3) cerdas plumosas. Exopodito (escafognatito) com 57 a 64 cerdas marginais plumosas e de 10 a 12 cerdas lisas e plumosas na região mediana.

Primeiro Maxilipodo (Fig. 3a). Endito coxal com 19 a 21 cerdas lisas e plumosas. Endito basal com 18 a 23 cerdas lisas e plumosas. Endopodito com três a oito cerdas lisas apicais e bulbo próximo a região distal com zero a duas cerdas 

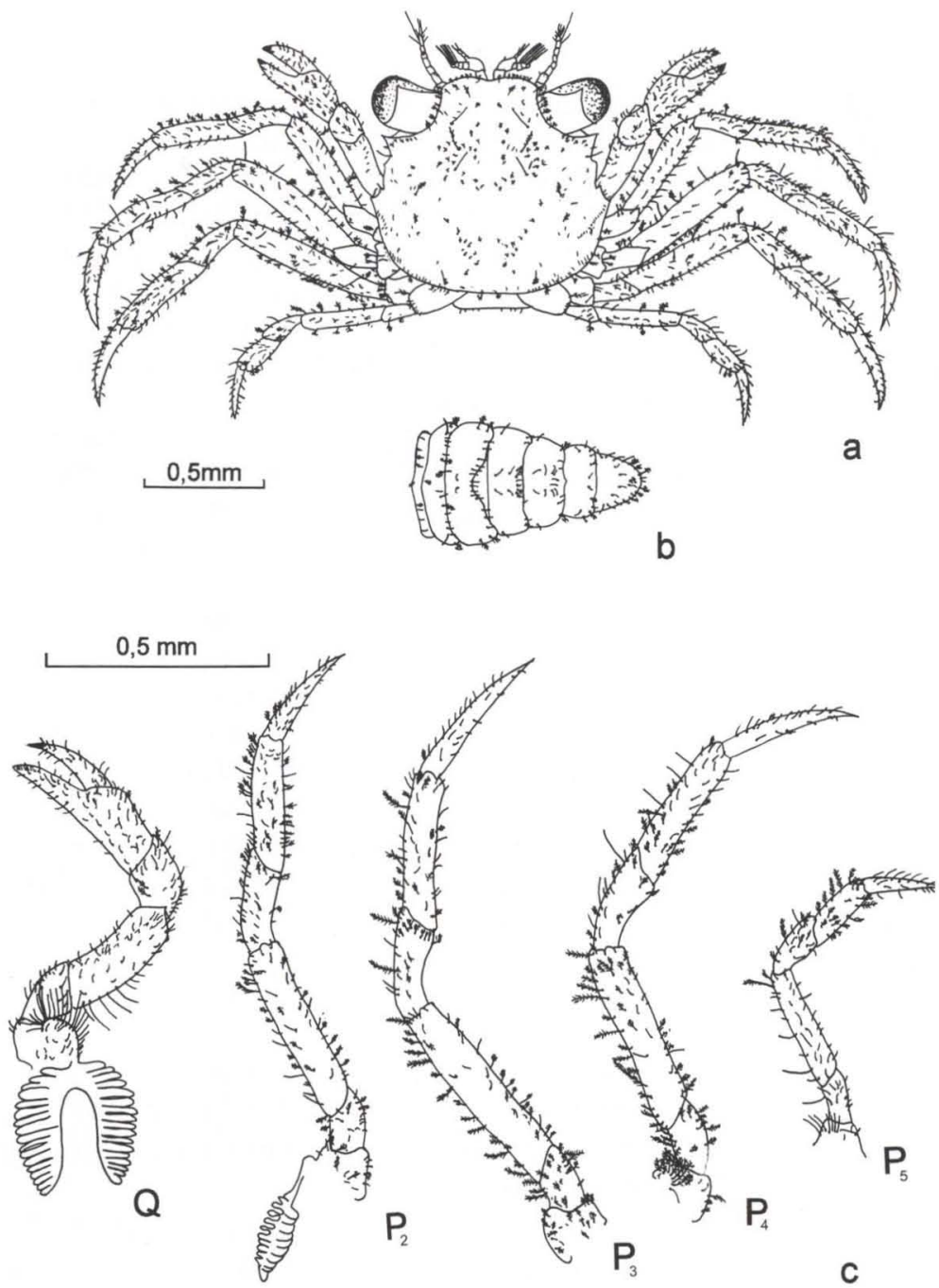

Fig. 1. Cyrtograpsus angulatus, juvenil I. (a)Abdômen; (b) quelipodo (Q); (c) segundo ao quinto pereiópodos (P2-P5).

plumosas. Exopodito com 5 (4) cerdas plumosas apicais na região distal e três a cinco cerdas plumosas e serrilhadas na região proximal. Epipodito bem desenvolvido com 17 a 21 cerdas longas lisas e plumosas com inserção supra cuticular.

Segundo Maxilípodo (Fig. 3b). Endopodito dividido em cinco segmentos providos de duas cerdas plumosas, três ou quatro cerdas plumosas, uma cerda lisa, cinco a nove cerdas lisas, plumosas e serrilhadas, 10 a 14 cerdas lisas, plumosas, serrilhadas e plumodenticuladas, respectivamente, do segmento proximal ao distal. Exopodito bi-segmentado com 15 (10) cerdas plumosas e serrilhadas no segmento 

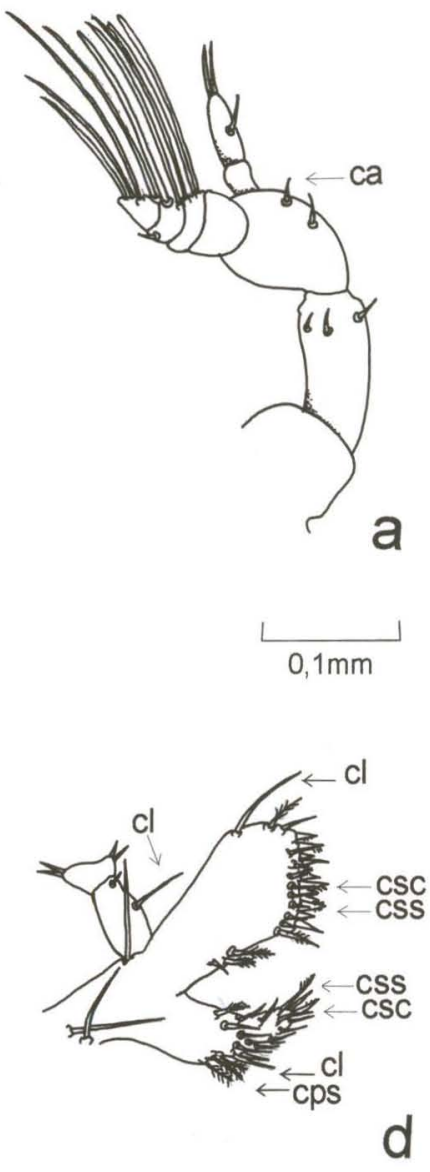

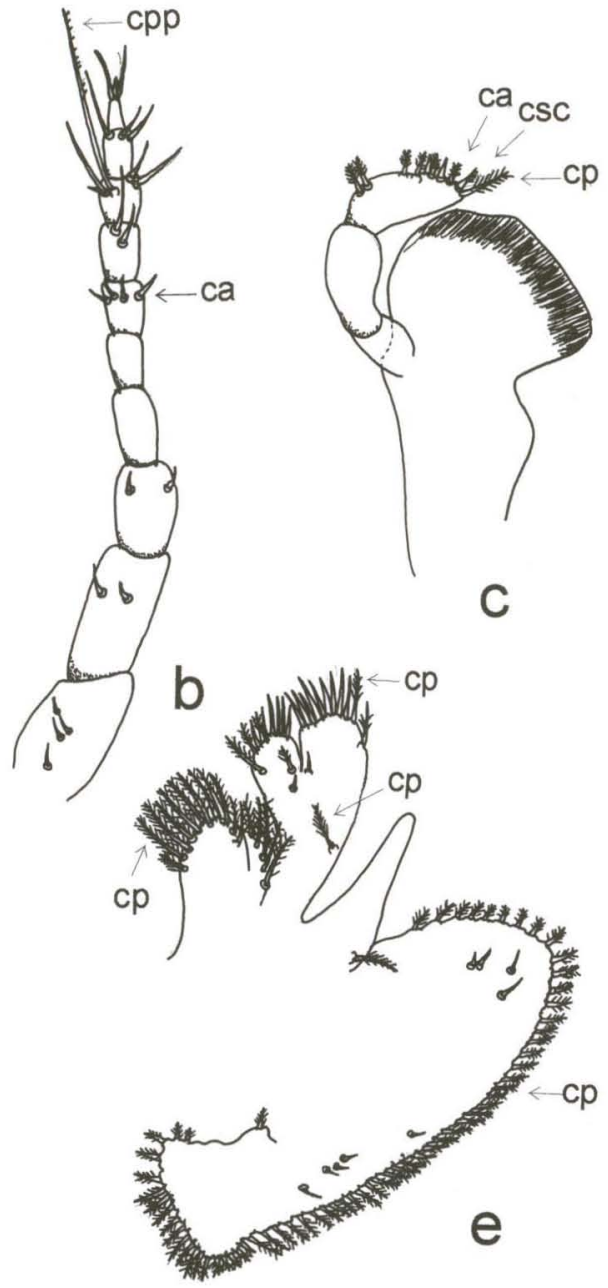

Fig. 2. Cyrtograpsus angulatus, primeiro estágio juvenil. (a) Antênula; (b) antena; (c) mandibula; (d) maxilula; (e) maxila.

proximal e 5 (6) cerdas apicais plumosas com inserção supra cuticular no segmento distal. Epipodito com três a cinco cerdas lisas e plumosas. Na base do epipodito diferencia-se uma pequena podobrânquia desprovida de lamelas.

Terceiro Maxilípodo (Fig. 3c). Endopodito com cinco segmentos providos de 25 a 31 cerdas lisas e plumosas, 15 ou 17 cerdas lisas e plumosas, 8 a 10 cerdas lisas e plumosas, 10 cerdas lisas, plumosas e serrilhadas, e 10 ou 11 cerdas lisas, plumosas, serrilhadas e plumodenticuladas, respectivamente, do segmento proximal ao distal. Exopodito bi-segmentado apresentando 6 (5) cerdas apicais plumosas com inserção supra cuticular no segmento distal e 17 (16) cerdas lisas, plumosas e serrilhadas no segmento proximal. Epipodito apresenta 40 a 48 cerdas plumosas na 
região basal e 49 a 54 cerdas lisas na região distal. Junto ao epipodito aparece uma artrobrânquia bem desenvolvida, a segunda artrobrânquia do apêndice aparece a partir do segundo estágio juvenil.

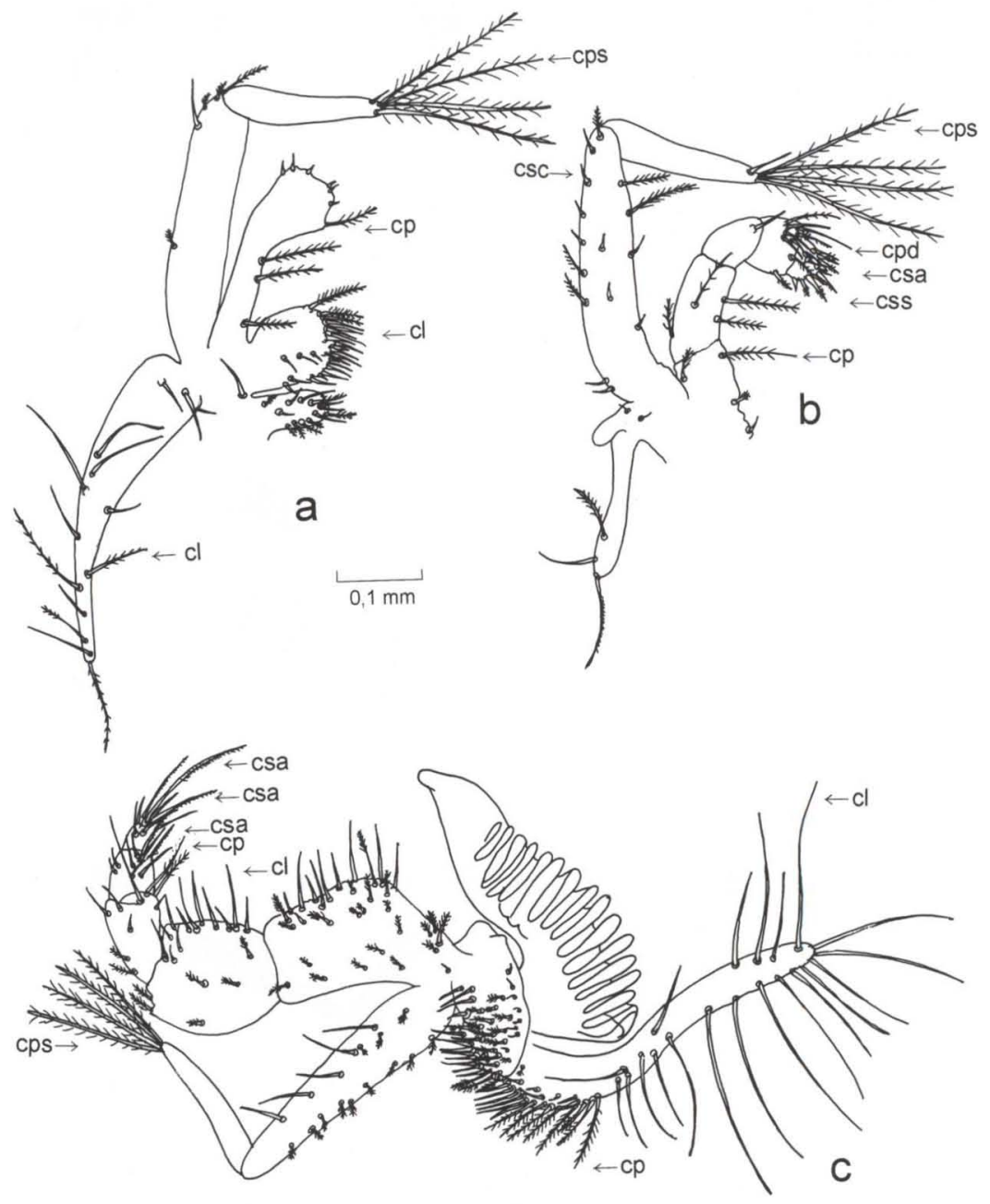

Fig. 3. Cyrtograpsus angulatus, primeiro estágio juvenil. (a) Primeiro maxilipodo; (b) segundo maxilípodo; (c) terceiro maxilipodo.

\section{Segundo ao oitavo estágios juvenis (Figs 4-10)}

A partir do segundo estágio juvenil verificou-se que os animais começam a apresentar uma série de modificações de ordem morfológica, embora, aparentemente, mantenham-se semelhantes no aspecto geral. Esta diferenciação acentua-se, principalmente, pela manifestação dos caracteres sexuais secundários. 


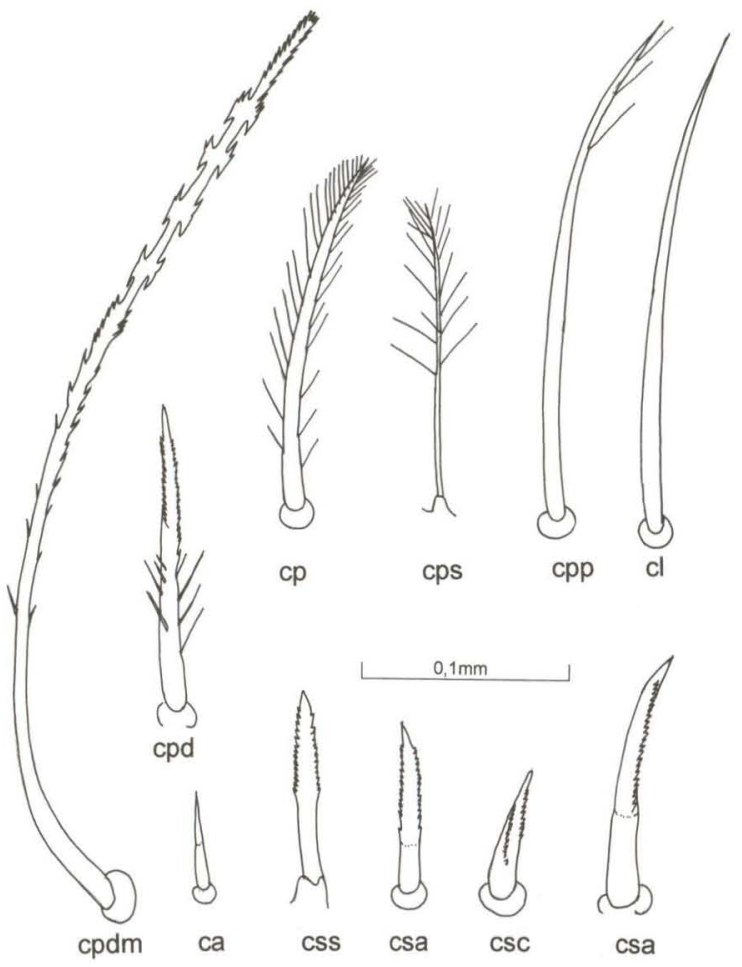

Fig. 4. Cyrtograpsus angulatus, diferentes tipos de cerdas presentes nas suas formas juvenis. (cp) Cerda plumosa; (cps) cerda plumosa com inserção supracuticular; (cpp) cerda pouco plumosa do ante-penúltimo segmento da antena; (cl) cerda lisa; (cpd) cerda plumodenticulada; (cpdm) cerda plumodenticulada com dentículos modificados em forma de bigorna "anvio-shaped denticules"; (ca) cerdas simples com "annulus"; (css) cerda serrilhada sem "annulus" e com inserção supracuticular; (csa) cerda serrilhada com "annulus"; (csc) cerda serrilhada com forma "cuspidate".

A carapaça, apresenta-se mais longa do que larga, praticamente quadrada, no primeiro estágio juvenil. A partir do segundo estágio juvenil passa a apresentar um formato ligeiramente mais largo do que longo, assemelhando-se ao que ocorre com os adultos. (Tab. II).

Tabela II. Cyrtograpsus angulatus. Medidas $(\mathrm{mm})$ do comprimento e largura da carapaça, do I ao VIII estágios juvenis. (L/C) Razão entre largura e comprimento.

\begin{tabular}{|c|c|c|c|c|c|c|c|}
\hline \multirow[t]{2}{*}{ Estágio } & \multicolumn{2}{|c|}{ Minimo } & \multicolumn{2}{|c|}{ Máximo } & \multicolumn{3}{|c|}{ Média } \\
\hline & Comprimento & Largura & Comprimento & Largura & Comprimento & Largura & $L / C$ \\
\hline $\mathrm{Jl}$ & 1,450 & 1,400 & 1,600 & 1,625 & 1,510 & 1,490 & 0,987 \\
\hline JII & 1,750 & 2,000 & 2,000 & 2,250 & 1,908 & 2,100 & 1,101 \\
\hline JIII & 2,150 & 2,560 & 2,730 & 3,230 & 2,572 & 2,982 & 1,159 \\
\hline JIV & 3,180 & 3,690 & 3.650 & 4,300 & 3,402 & 3,999 & 1,175 \\
\hline JV & 3,460 & 4,000 & 4,460 & 5,150 & 3,927 & 4,626 & 1,178 \\
\hline JVI & 3,920 & 4,690 & 4,960 & 6,000 & 4,693 & 5,539 & 1,180 \\
\hline JVII & 4,380 & 5,230 & 5,380 & 6,530 & 4,981 & 5,925 & 1,189 \\
\hline JVIII & 5,380 & 6.460 & 6.530 & 7,660 & 5.880 & 7,000 & 1.190 \\
\hline
\end{tabular}




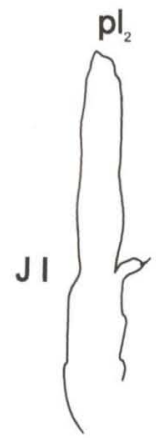

$\mathrm{pl}_{3}$

$\mathrm{pl}_{4}$

$\mathrm{pl}_{5}$

U
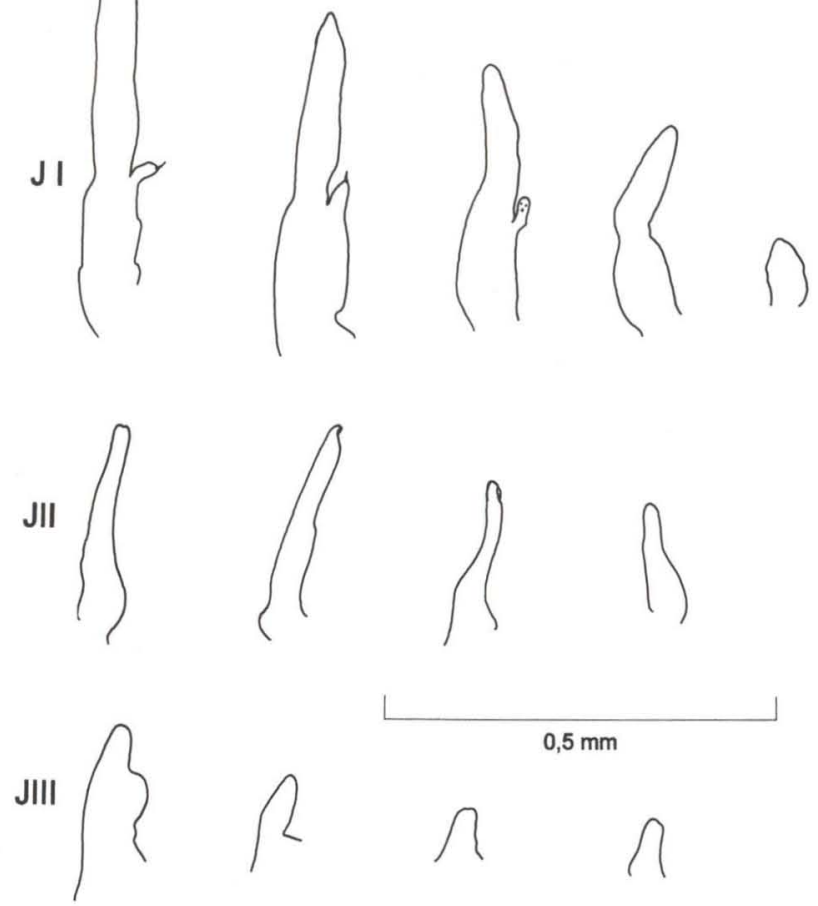

$0,5 \mathrm{~mm}$

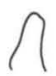

Fig. 5. Cyrtograpsus angulatus, pleópodos do primeiro ao terceiro estágios juvenis.

Em Chasmagnathus granulata, esta inversão da relação comprimento largura ocorre no quinto estágio juvenil (RIEGER \& NAKAGAWA 1995). Em Sesarma (Holometopus) rectum, isto ocorre no sexto estágio juvenil (FrANSOZO 1986). Com relação à ontogenia branquial, em Cyrtograpsus angulatus as brânquias se apresentam completas em número a partir do segundo estágio juvenil, enquanto Chasmagnathus granulata apresenta o seu número completo no primeiro estágio juvenil.

No que diz respeito à diferenciação dos caracteres sexuais secundários, verificou-se que as modificações mais acentuadas ocorreram nos pleópodos, cuja evolução é apresentada nas figuras 5 a 10 . O primeiro estágio apresenta quatro pares de pleópodos e um par de urópodo. Os três primeiros pares são providos de um pequeno bulbo, nos quais aparecem pequenas cerdas, o ultimo par e o urópodo são desprovidos de bulbos ou cerdas. No segundo estágio ocorre o desaparecimento dos bulbos e das cerdas nos três primeiros pares, ocorrendo uma diminuição de tamanho de todos os pleópodos além do desaparecimento dos urópodos. No terceiro estágio os pleópodos aparecem de forma rudimentar e de tamanho bem reduzido. No quarto estágio ocorre o dimorfismo sexual. Nos machos, aparece um novo par de pleópodos, provido de cerdas, no primeiro segmento abdominal, e o desaparecimento dos três últimos pares de pleópodos, mantendo-se então os pleópodos correspondentes 
ao primeiro e segundo segmentos abdominais. Do quinto ao oitavo estágios, sofrem apenas aumento de tamanho e do número de cerdas, sendo que o primeiro par de pleópodo, a cada estágio, fica proporcionalmente maior do que o segundo par, conforme observado no adulto.

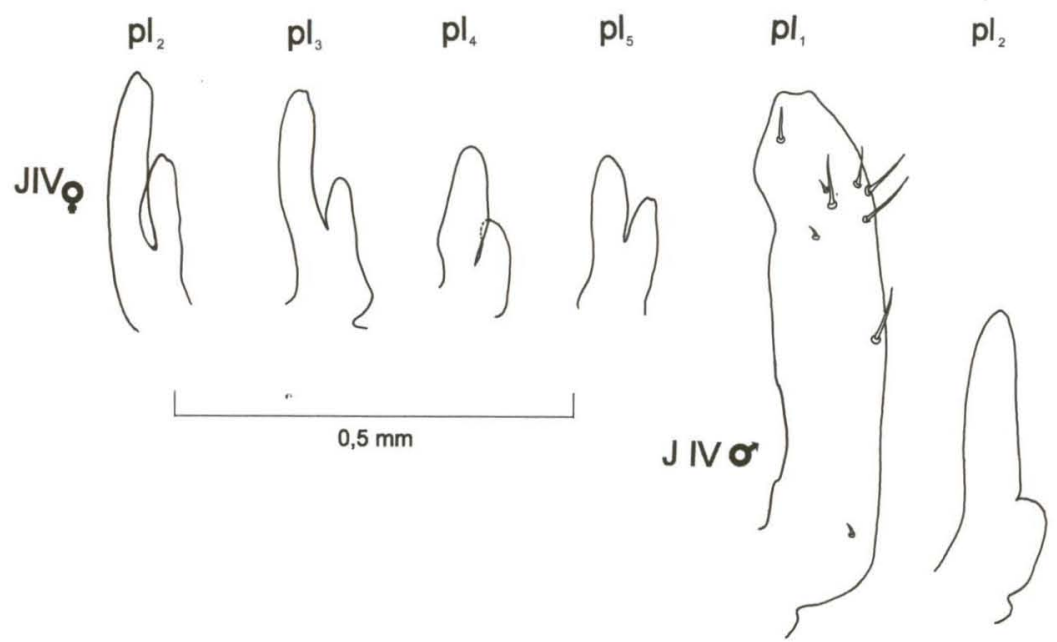

Fig. 6. Cyrtograpsus angulatus, pleópodos do quarto estágio juvenil.

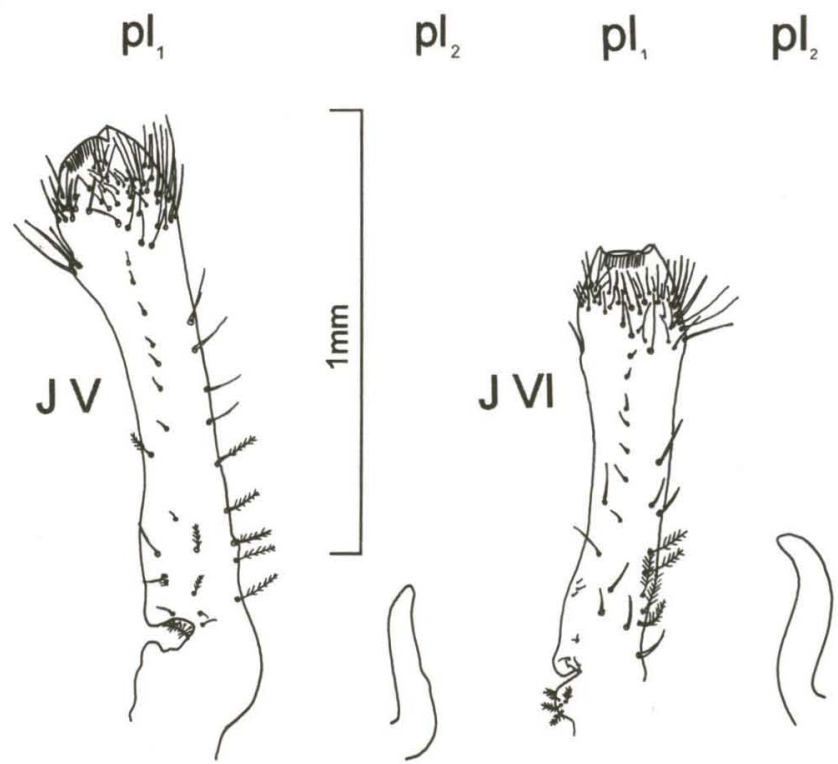

Fig. 7. Cyrtograpsus angulatus, pleópodos das fêmeas do quinto e sexto estágios juvenis. 
Já nas fềmeas, a partir do quarto estágio, são observados quatro pleópodos birremes, do segundo ao quinto segmento abdominal, sendo que estes são desprovidos de cerdas e de tamanho inferior aos do macho. No quinto estágio estes sofrem um aumento de tamanho, e ocorre o surgimento de cerdas distribuídas ao longo dos mesmos. A partir do sexto estágio passam a apresentar segmentação no endopodito, além do aumento de tamanho e do número de cerdas. No sétimo e oitavo estágios sofrem aumento de tamanho, número de cerdas e tamanho de cerdas.

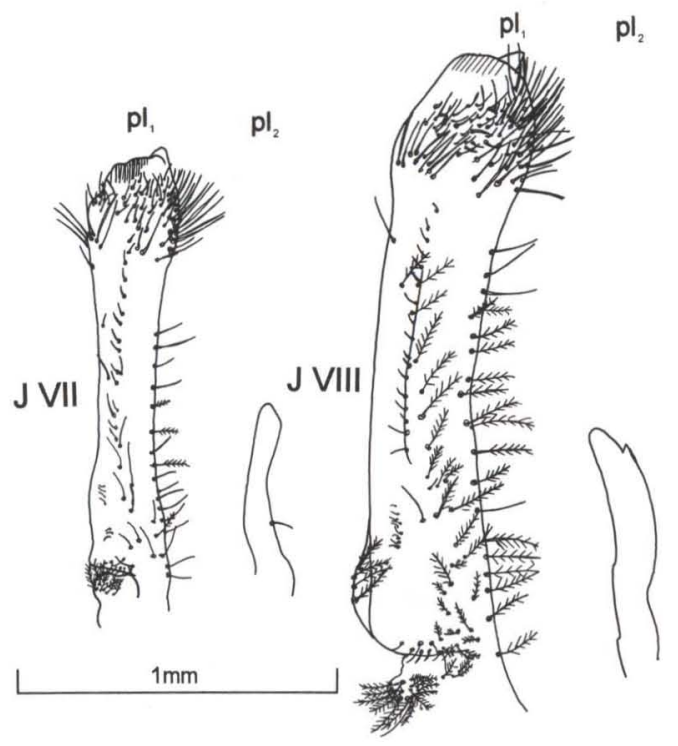

Fig. 8. Cyrtograpsus angulatus, pleópodos das fêmeas do sétimo e oitavo estágios juvenis.
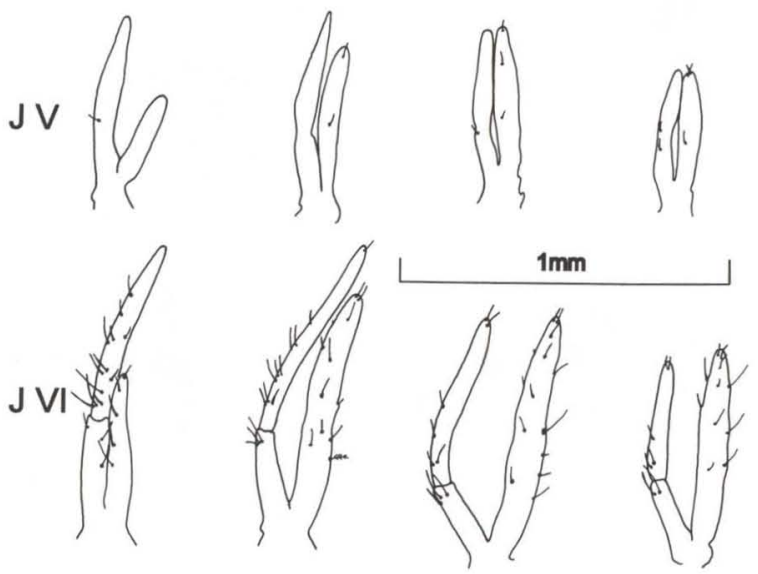

Fig. 9. Cyrtograpsus angulatus, pleópodos dos machos do quinto e sexto estágios juvenis. 


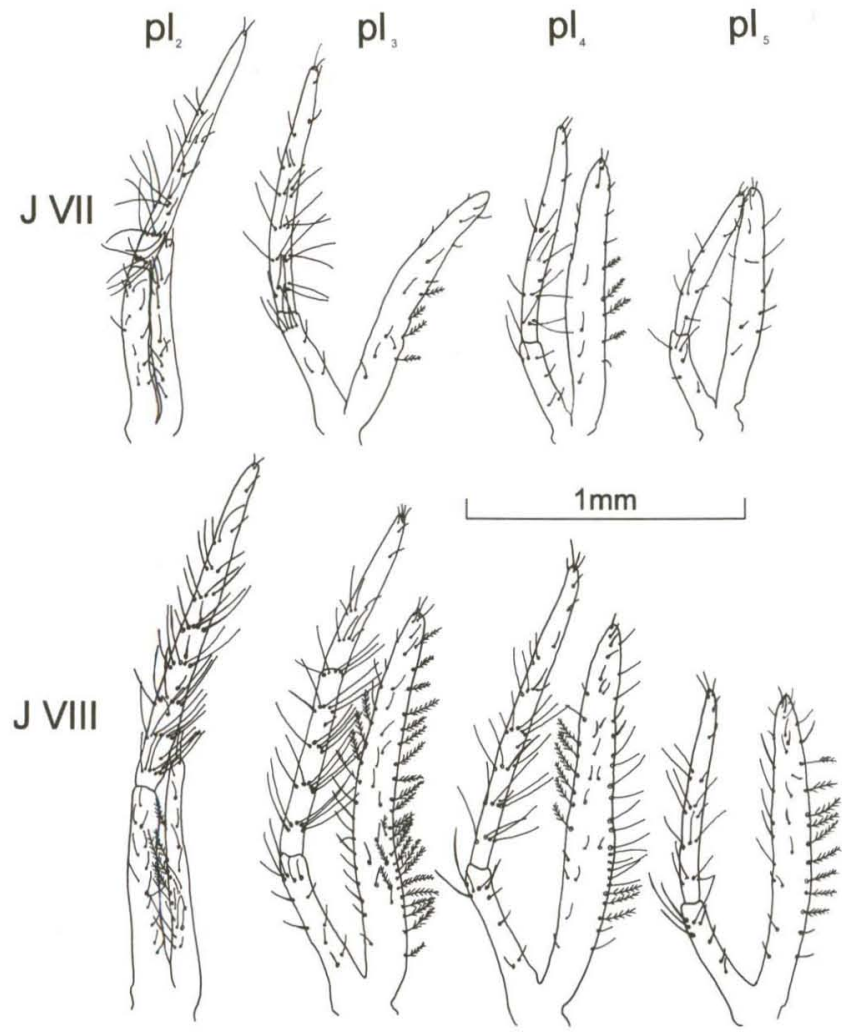

Fig. 10. Cyrtograpsus angulatus, pleópodos dos machos do sétimo e oitavo estágios juvenis.

Em Chasmagnathus granulata o dimofismo sexual ocorre a partir do terceiro estágio e, em Pachygrapsus transversus (FLORES et. al. 1998) o dimorfismo sexual ocorre no segundo estágio juvenil. Já em Sesarma rectum (FRANSOZO 1986) ocorre no décimo segundo estágio e, em Panopeus herbstii H. Milne-Edwards, 1834 estudado por HEBLING et al. (1982) o dimorfismo, ocorre no quarto estágio.

Ainda com relação aos caracteres sexuais secundários, observou-se uma considerável modificação no formato do abdômen a partir do quarto estágio, tornando-se mais acentuada no oitavo estágio. $\mathrm{O}$ abdômen das fêmeas vai se tornando gradativamente arredondado, enquanto o dos machos adquire um formato retangular. Também foi possível observar o surgimento de cerdas modificadas no epipodito do primeiro maxilípodo, do tipo plumodenticuladas, longas com dentículos modificados com formato de bigorna.

Para a diferenciação dos estágios juvenis, os caracteres de maior valor prático, até o oitavo estágio, e que permitem sua respectiva identificação, estão representados na tabela III.

Uma comparação entre caracteres juvenis da família Grapsidae pode ser observada na tabela IV. 
Tabela III. Cyrtograpsus angulatus. Principais caracteres morfológicos do segundo (JII) ao oitavo (JVIII) estágios juvenis. (EX) Exopodito, (EN) endopodito, $(B)$ base, (CO) coxa, (AU) antênula, (AN) antena, (MXU) maxilula, (MXA) maxila, (MP) mandibula. Os números ou intervalos que ocorreram em menor freqüência estão entre parênteses.

\begin{tabular}{|c|c|c|c|c|c|c|c|}
\hline Caráter & JII & JIII & JIV & JV & JVI & JVII & JVIII \\
\hline Segmentos do EX da $\mathrm{AU}$ & $4(3)$ & $4(5)$ & 5 & $6(5)$ & 6 & $7(6)$ & $7(8)$ \\
\hline Segmentos do EN da AU & 2 & 2 & 2 & 2 & 2 & 2 & 2 \\
\hline Cerdas do EN da AU primeiro segmento & 0 & 0 & 0 & 1 & 1 & $1(2)$ & $1(2)$ \\
\hline Cerdas do EN da AU segundo segmento & $4(3)$ & $4(5)$ & $5(6)$ & $6(7,8)$ & $9(6,8)$ & $9(7,8)$ & $8(7,9)$ \\
\hline Segmentos da AN & 10 & $10(9)$ & $11(12)$ & $13(11,12)$ & $12(12)$ & $13(12)$ & $13(15)$ \\
\hline Cerdas do segmento basal do EN da MXU & $2(1,3)$ & $2(3)$ & $3(2,4)$ & $4(3,5)$ & $5(6)$ & $6(5,7,8)$ & $7(6,8)$ \\
\hline Cerdas do EN da MXU & $6(5,8)$ & $8(7,9)$ & $10(6$ a 12$)$ & $10(7$ a 12$)$ & $11(12)$ & $11(10$ a 13$)$ & $13(12,15)$ \\
\hline Cerdas do B da MXU & $31(28$ a 31$)$ & 30 a 38 & $45(41$ a 49$)$ & $54(55,56)$ & $64(64$ a 72$)$ & 70 a 82 & 77 a 96 \\
\hline Cerdas do CO da MXU & $30(23$ a 30$)$ & $36(33$ a 41$)$ & $62(54$ a 65$)$ & 78 (71 a 98$)$ & $96(80$ a 106$)$ & $113(103$ a 121$)$ & 103 a 133 \\
\hline Cerdas marginais do EX da MXA & $70(98$ a 89$)$ & $105,108(95$ a 110$)$ & $140(126$ a 158$)$ & 147 a 207 & 171 a 227 & $222(200$ a 234) & 239 (201 a 259) \\
\hline Cerdas do EN da MXA & $3(2$ a 4$)$ & $3(2)$ & $4(3,5)$ & $5(3,4,6)$ & $6(4$ a 7$)$ & $7(6,9)$ & $7(5,6,8)$ \\
\hline Cerdas do lobo distal do B da MXA & $16(15$ a 19$)$ & $21(21$ a 24$)$ & $33(30$ a 39$)$ & $45(42$ a 52$)$ & $50(46$ a 62$)$ & $58(54$ a 62$)$ & $63(57$ a 68$)$ \\
\hline Cerdas do lobo proximal do B da MXA & $13(14)$ & $(16$ a 23) & $24,25$ ( 23 a 28$)$ & $32(24$ a 39$)$ & $35(28$ a 38$)$ & 33 a 40 & $44(40$ a 52$)$ \\
\hline Cerdas do lobo distal do CO da MXA & $8,9(7$ a 10$)$ & $11(10$ a 12$)$ & $14(13$ a 15$)$ & $16(13$ a 23$)$ & 17 a 20 & $20(20$ a 23$)$ & $23,24$ (20 a 27$)$ \\
\hline Cerdas do lobo proximal do CO da MXA & $21(22)$ & $27,28$ (25 a 31$)$ & $36(33$ a 37$)$ & $47,50(43$ a 52$)$ & $52(48$ a 58$)$ & $60(56$ a 64$)$ & $64(57$ a 68$)$ \\
\hline Cerdas apicais do EN do primeiro maxilipodo & $7(4$ a 8$)$ & $(7$ a 10$)$ & $13(10$ a 14$)$ & $17(15$ a 23$)$ & $20(17$ a 21$)$ & $22(17$ a 22$)$ & $22(20$ a 22$)$ \\
\hline Cerdas do bulbo do primeiro maxilipodo & $5(4)$ & $7,8(4$ a 8$)$ & $14(10$ a 12$)$ & 19 (12 a 22$)$ & $28(21$ a 30$)$ & 35 (28 a 39) & $34(29$ a 38$)$ \\
\hline Cerdas apicais do EX do primeiro maxilipodo & 5 & $5(6)$ & $8(7,9)$ & $10(9,11)$ & $12(10,13)$ & $14(13)$ & $16(14,15)$ \\
\hline Cerdas do $\mathrm{B}$ do primeiro maxilipodo & 24 (21 a 32) & $35(32$ a 39$)$ & $54,55$ (44 a 55$)$ & $67,71$ (61 a 80$)$ & 72 a 98 & 89 a 108 & 98 a 115 \\
\hline Cerdas do $\mathrm{CO}$ do primeiro maxilipodo & $30(28$ a 33$)$ & $42(41$ a 48$)$ & $59(56$ a 59$)$ & $86(81$ a 96$)$ & 99 (95 a 11) & 101 a 126 & 129 a 158 \\
\hline Cerdas do quarto segmento do EN do segundo maxilipodo & $8,9(7)$ & $14(13$ a 15$)$ & $19(18,21,24)$ & $24(24$ a 32$)$ & $27(26$ a 34$)$ & 29 a 37 & $36(32$ a 41$)$ \\
\hline Cerdas do quinto segmento do EN do segundo maxilipodo & 11 a 15 & $16(13$ a 19$)$ & 18 a 23 & $24(24$ a 30$)$ & $28(27$ a 31$)$ & 30 a 36 & 35 a 42 \\
\hline Cerdas do último segmento do $\mathrm{EX}$ do segundo maxilipodo & $6(5)$ & $5(6)$ & 8 & $11(10,12)$ & $12(13,14)$ & $14(15,16)$ & $16(17,18,19)$ \\
\hline Cerdas do penúltimo segmento do EN do terceiro maxilipodo & $10,11(12)$ & $14(13,15,16)$ & $24(22,26,30)$ & 35 a 52 & 46 a 64 & 49 a 81 & 75 a 92 \\
\hline Cerdas do último segmento do EN do terceiro maxilipodo & $11(10,12)$ & $14(11,12,13)$ & 18,19 & 26 a 36 & $38(26$ a 43$)$ & 35 a 53 & 39 a 55 \\
\hline Cerdas do último segmento do EX do terceiro maxilipodo & $5(6)$ & $6(5)$ & $9(8,11,12)$ & $12(10,13,14)$ & $14(15)$ & $16(17)$ & $\begin{array}{l}17,18(16,19, \\
21)\end{array}$ \\
\hline Cerdas do MP no primeiro segmento & 0 & 0 & 0 & 0 & 0 & 0 & 0 \\
\hline Cerdas do MP no segundo segmento & 0 & $4(3)$ & $6(4,7,8)$ & $10(6,11,12)$ & $14(12,15,16)$ & $14,16,17(18)$ & $18(16,19,21)$ \\
\hline Cerdas do MP no terceiro segmento & 12 a 17 & $21(20$ a 25$)$ & $31(28$ a 32$)$ & 42 a 55 & 47 a 63 & $69(56$ a 82$)$ & 79 a 93 \\
\hline
\end{tabular}


Tabela IV. Comparação de caracteres juvenis da familia Grapsidae. Autores: (1) FUKUDA \& BabA 1976; (2, 3) Diaz \& EWAld 1968; (4) Fransozo 1986; (5) Rieger \& NaKagawa 1995; (6) ANGer et al. 1995; (7) PAULA \& HARTNOL 1989; (8) FLORES et al. 1998; (9) Presente trabalho.

\begin{tabular}{|c|c|c|c|c|c|c|c|}
\hline Espécie & Sub-Familia & A & B & C & D & $E$ & $\mathbf{F}$ \\
\hline Chiromantes bidens ( 1 ) & Sesarminae & & $x$ & $x$ & & $x$ & $x$ \\
\hline Armases rubripes (2) & Sesarminae & & $x$ & & $x$ & $x$ & $x$ \\
\hline Sesarma ricordi (3) & Sesarminae & & $x$ & & $x$ & $x$ & $x$ \\
\hline Sesarma rectum (4) & Sesarminae & & $x$ & & $x$ & $x$ & $x$ \\
\hline Chasmagnathus granulata $(5)$ & Sesarminae & $x$ & & & $x$ & & \\
\hline Sesarma curacaoense (6) & Sesarminae & Ausente & Ausente & & $x$ & $x$ & $\mathrm{x}$ \\
\hline Percnon gibbesi (7) & Plagusiinae & Ausente & Ausente & \multicolumn{4}{|c|}{ Năo descrito Năo descrito Năo descrito Năo descrito } \\
\hline Pachygrapsus transversus ( 8 ) & Grapsinae & Ausente & Ausente & \multicolumn{4}{|c|}{$x$} \\
\hline Cyrtograpsus angulatus (9) & Varuninae & $\mathrm{x}$ & & \multicolumn{4}{|c|}{$\mathrm{x}$} \\
\hline
\end{tabular}

(A)Cerda longa no antipenúltimo segmento da antena; (B) cerda longa no penúltimo segmento da antena; (C) palpo da mandibula tri-segmentado; (D) palpo da mandíbula bi-segmentado; (E) ausência de epipodito do segundo maxilipede; (F) ausência de endopodito da antênula; (X) presença de estruturas.

AGRADECIMENTOS. Os autores agradecem o subsídio oferecido pela FAPERGS através do processo $\mathrm{N}^{\circ} 95 / 51017.5$ è̀s pessoas envolvidas direta ou indiretamente no trabalho, em especial a Sandro Klippel pelo apoio e sugestões.

\section{REFERÊNCIAS BIBLIOGRÁFICAS}

ANGER, K.; D. SCHREIBER \& M. Montú. 1995. Abbreviated larval development of Sesarma curacaoense (Rathbun, 1897) (Decapoda: Grapsidae) reared in the laboratory. Nauplius 3: 127-154.

BABA, K. \& K. MiyatA. 1971. Larval development of Sesarma (Holometopus) dehaani H. Milne Edwards (Crustacea, Brachyura) reared in the laboratory. Mem. Fac. Educ. Kumamoto Univ. 19: 54-64.

Baba, K. \& M. Moriyama. 1972. Larval development of Helice tridens wuana Rathbun and $H$. tridens de Haan (Crustacea, Brachyura) reared in the laboratory. Mem. Fac. Educ. Kumamoto Univ. 20: 49-68.

Bond-Buckup, G.; A.A. Bueno \& K.A. Keunecke. 1996. Primeiro estágio juvenil de Aegla prado Schmitt (Crustacea, Decapoda, Anomura, Aeglidae). Revta bras. Zool. 13: 1049-1061.

DIAZ, H. \& J.J. EWALD. 1968. A comparison of the larval development of Metasesarma rubripes (Rathbun) and Sesarma ricordi H. Milne Edwards (Brachyura, Grapsidae) reared under similar laboratory conditions. Crustaceana (Suppl. 2): 225-248.

DRACH, P. \& F. JACQUES. 1977a. Système sétifère des crustacés décapodes. Le système microsétal. C.R. Acad. Sc. Paris 284: 1103-1105.

1977b. Système sétifère des crustacés décapodes. Principes d'une classification générale. C.R. Acad. Sc. Paris 284: 1995-1998.

FACTOR, J. 1978. Morphology of the mouthparts of larval lobsters, Homarus americanus (Decapoda: Nephropidae), with special emphasis on their setae. Biol. Bull. 154: 383-408.

FARMER, A.S. 1974. The functional morphology of the mouthparts and pereiopods of Nephrops norvegicus (L.) (Decapoda: Nephropidae). Jour. Nat. Hist. 8: 121-142.

Fish, S. 1972. The setae of Eurydice pulchra (Crustacea: Isopoda). Jour. Zool., London, 166: 163-177.

Flores, A.A. V.; M.L. Negreiros Fransozo \& A. Fransozo. 1998. The megalopa and juvenile development of Pachygrapsus transversus (Gibbes, 1850) (Decapoda, Brachyura), compared with other grapsid crabs. Crustaceana 71 (2): 197-222.

Fransozo, A. 1986. Desenvolvimento dos estágios juvenis de Sesarma (Holometopus) rectum Randall, 1840 (Decapoda, Grapsidae) obtidos em laboratório. Naturalia 11/12: 77-87. 
Fukuda, Y. \& K. BABA. 1976. Complete larval development of the Sesarminid crabs, Chiromantes bidens, Holometopus haematocheir, Parasesarma plicatum and Sesarmops intermedius, reared in the laboratory. Mem. Fac. Educ. Kumamoto Univ. 25: 61-75.

HartNoll, R.G. 1992. Megalopae and early postlarval stages of East African Percnon (Decapoda: Brachyura: Grapsidae). Jour. Zool., London, 228: 51-67.

Hebling, N.J.; A. Fransozo \& M.L. Negreiros-Fransozo. 1982. Desenvolvimento dos primeiros estágios juvenis de Panopeus herbstii H. Milne-Edwards, 1834 (Crustacea, Decapoda, Xanthidae), criados em laboratório. Naturalia 7: 177-188.

INGLE, R. 1992. Larval Stages of Northeastern Atlantic Crabs. London, Chapman \& Hall, 363p.

JACQues, F.; L. WATLING \& A.B. ThistLl. 1989. The setal system of crustaceans: Types of setae, groupings, and functional morphology, p. 1-13. In: B.E. Felgenhauer (Ed.). Functional Morphology of Feeding and Grooming in Crustacea. Crustacean Issues 6. Rotterdam, A.A. Balkema, 221p.

KUNZE, J. \& D.P. ANDERSON. 1979. Functional morphology of the mouthparts and gastric mill in the hermit crabs Clibanarius taeniatus (Milne Edwards), Clibanarius virescens (Krauss), Paguristes squamosus (McCulloch) and Dardanus setifer (Milne Edwards) (Anomura: Paguridae). Aust. Jou. Mar. Freshw. res. 30: 683-722.

LAM, S.C. 1969. Abbreviated development of a non-marine crab, Sesarma (Geosesarma) perracae (Brachyura, Grapsidae) from Singapore. Jour. Zool., London, 158: 351-370.

Melo, G.A.S. 1996. Manual de Identificação dos Brachyura (Caranguejos e Siris) do Litoral Brasileiro. São Paulo, Ed. Plêiade, 604p.

Paula, J. \& R.G. Hartnoll. 1989. The larval and post-larval development of Percnon gibbesi (Crustacea, Brachyura, Grapsidae) and the identity of the larval genus Pluteocaris. Jour. Zool. 218: 17-37.

PoHLE, G. \& M. TEl.Ford. 1981. Morphology and classification of decapod crustacean larval setae: a scanning electron microscope study of Dissodactylus crinitichelis Moreira, 1901 (Brachyura: Pinnotheridae). Bull. Mar. Sci. 31: 736-752.

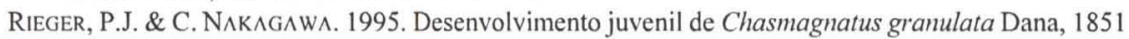
(Crustacea, Decapoda, Grapsidae), em laboratório. Nauplius 3: 59-74.

Rieger, P.J. \& R.R. VieiRA. 1997. Desenvolvimento larval de Cyrtograpsus angulatus Dana, 1851 (Crustacea, Decapoda, Grapsidae) em laboratório. Revta bras. Zool. 14 (3): 601-623.

Rodriguez, A.; J.I. GonZALFz-Gordillo \& J.A. CuFsTA. 1992. Larval stages of Brachynotus atlanticus Forest, 1957 (Crustacea: Decapoda: Grapsidae) reared under laboratory conditions. Jour. Plank. Res. 14: 867-883.

SCHEMBRI, P.J. 1982. Functional morphology of the feeding and grooming appendages of Ebalia tuberosa (Pennant) (Crustacea: Decapoda: Leucosiidae). Jour. Nat. Hist. 16: 467-480.

Тном^s, W.J. 1970. The setae of Austropotamobius pallipes (Crustacea: Astacidae). Jour. Zool. 160: 91-142.

Thompson, M.T. 1903. The Metamorphose of Hermit Crab. Proc. Boston Soc. Nat. Hist. 31: 147-209.

WATLING, L. \& A.B. ThisTLE. 1989. A classification system for crustacean setae based on the homology concept, p. 15-26. In: B.E. Ffigenhauer (Ed.). Functional Morphology of Feeding and Grooming in Crustacea. Crustacean Issues 6. Rotterdam, A.A. Balkema, 221p.

Recebido em 22.I.1999; aceito em 18.IV.2000 